\title{
Super-cool Dark Matter
}

\author{
Thomas Hambye, ${ }^{a}$ Alessandro Strumia ${ }^{b, c, d}$ and Daniele Teresi ${ }^{a}$ \\ ${ }^{a}$ Service de Physique Théorique, Université Libre de Bruxelles, \\ Boulevard du Triomphe CP225, 1050 Brussels, Belgium \\ ${ }^{b}$ CERN, Theory Division, \\ Geneva, Switzerland \\ ${ }^{c}$ Dipartimento di Fisica "E. Fermi", Università di Pisa, \\ Largo Bruno Pontecorvo 3, I-56127 Pisa, Italy \\ ${ }^{d}$ INFN, Sezione di Pisa, \\ Edificio C-Polo Fibonacci, Largo Bruno Pontecorvo 3, I-56127 Pisa, Italy \\ E-mail: thambye@ulb.ac.be, Alessandro.Strumia@cern.ch, \\ Daniele.Teresi@ulb.ac.be
}

ABStRACT: In dimension-less theories of dynamical generation of the weak scale, the Universe can undergo a period of low-scale inflation during which all particles are massless and undergo super-cooling. This leads to a new mechanism of generation of the cosmological Dark Matter relic density: super-cooling can easily suppress the amount of Dark Matter down to the desired level. This is achieved for TeV-scale Dark Matter, if super-cooling ends when quark condensates form at the QCD phase transition. Along this scenario, the baryon asymmetry can be generated either at the phase transition or through leptogenesis. We show that the above mechanism takes place in old and new dimension-less models.

Keywords: Beyond Standard Model, Cosmology of Theories beyond the SM, Higgs Physics, Spontaneous Symmetry Breaking

ARXIV EPRINT: 1805.01473 


\section{Contents}

1 Introduction 1

2 General mechanism 2

2.1 Super-cooling 3

2.2 Reheating 3

2.3 The phase transition to the massive vacuum 5

2.4 The baryon asymmetry 5

3 Model with $\mathrm{SU}(2)_{X}$ gauge group $\quad 6$

$\begin{array}{lll}3.1 & \text { Super-cooling } & 7\end{array}$

$\begin{array}{ll}3.2 \text { End of super-cooling } & 7\end{array}$

3.3 Reheating 9

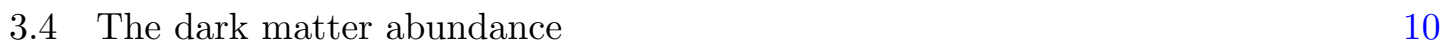

$\begin{array}{ll}3.5 & \text { The baryon asymmetry } \\ \end{array}$

4 Model with $\mathrm{U}(1)_{B-L}$ gauge group 13

$\begin{array}{lll}5 & \text { Summary } & 16\end{array}$

\section{Introduction}

Dark Matter $(\mathrm{DM})$ could be particles with mass $M_{\mathrm{DM}} \gg \mathrm{keV}$. The cosmological DM abundance $\Omega_{\mathrm{DM}} h^{2} \approx 0.110$ is reproduced if their number density in units of the entropy density $s$ is small:

$$
\frac{n_{\mathrm{DM}}}{s}=\frac{0.40 \mathrm{eV}}{M_{\mathrm{DM}}} \frac{\Omega_{\mathrm{DM}} h^{2}}{0.110} .
$$

When DM becomes non-relativistic, thermal freeze-out at $T \sim T_{\text {dec }} \approx M_{\mathrm{DM}} / 25$ leaves the DM abundance $n_{\mathrm{DM}} / s \sim 1 /\left(M_{\mathrm{DM}} M_{\mathrm{Pl}} \sigma_{\mathrm{ann}}\right)$, where $\sigma_{\mathrm{ann}}$ is the $\mathrm{DM}$ annihilation cross section. As well known, $M_{\mathrm{DM}} \sim \mathrm{TeV}$ and $\sigma_{\mathrm{ann}} \sim 1 / M_{\mathrm{DM}}^{2}$ reproduces the desired DM abundance. Many alternative cosmological DM production mechanisms are possible, sometimes at the price of increasing model-building complexity.

We here discuss a new mechanism that can generate the desired cosmological DM abundance. The new mechanism is characteristic of models where a scale (we will consider the weak scale) is dynamically generated from a quantum field theory that only has dimension-less couplings. We assume that, in this context, all particles (in particular dark matter and the Higgs boson) remain massless until a vacuum expectation value or condensate develops. In the case of scalars, this conjecture is at odds with the usual view that attributes physical meaning to power-divergent quantum corrections, leading to the expectation that the Higgs boson should have been accompanied by new physics able of keeping its mass naturally much smaller than the Planck mass, the presumed cut-off of quantum field theories. The observation of the Higgs boson not accompanied by any new physics promoted renewed interest in dimension-less dynamics as the possible origin of the 
weak scale, see e.g. [1-5], and in attempts of building weak-scale extensions of the Standard Model valid up to infinite energy, such that no cut-off is needed [6-9]. More generically, super-cooling takes place if, for whatever reason, the mass scales in the potential are much smaller than the scale generated through dynamical transmutation. If small scalar masses are unnatural, super-cooling is a cosmological signature of such unnaturalness.

The mechanism relies on the fact that in dimension-less models, along its thermal history, the Universe remains trapped for a while in a phase of thermal inflation during which all particles are massless, so that DM undergoes super-cooling rather than freeze-out. The formation of QCD condensates at the QCD phase transition ends this phase, leading to the electro-weak phase transition, i.e. particle mass generation. From this point particles lighter than the reheating temperature can easily thermalize, but DM will not necessarily thermalize, leading to the necessary suppression of the DM relic density, provided that its mass is at the $\mathrm{TeV}$ scale. In the context of freeze-out, the same coincidence is advertised as 'WIMP miracle'.

In section 2 we present the mechanism. In the next sections we consider specific models, pointing out that no ad hoc model building is needed. Indeed, in section 3 we consider the model proposed in [10] (for the non scale invariant version of this model see [11-13]), that extends the SM by adding a scalar doublet under a new $\mathrm{SU}(2)$ gauge group (its vectors are automatically stable DM candidates). We find that this model can reproduce the observed DM either through freeze-out (at larger values of the gauge coupling [10]) or through supercooling (at smaller values of the gauge coupling). Super-cooling erases the baryon asymmetry: we will discuss how it can be regenerated at the weak scale, possibly through leptogenesis. Motivated by leptogenesis and neutrino masses, in section 4 we propose a similar model with $\mathrm{U}(1)_{B-L}$ gauge group and two extra scalars. Conclusions are given in section 5 .

While we focus on simple models based on weakly-coupled elementary particles, supercool DM can also arise in more generic contexts, such as strongly coupled models with walking dynamics [14], possibly described through broken conformal symmetries and/or through branes in warped extra dimensions [15]. In such a case super-cooling can be described geometrically [16]. Furthermore, super-cool DM could arise in extensions of the Standard Model that cut quadratically divergent corrections to the Higgs mass and generate the weak scale: for example in supersymmetric models where all particles are massless in the supersymmetric limit, and where supersymmetry gets dynamically broken by some expectation value. However, the non-observation of new physics at the Large Hadron Collider casts doubts on such models. In more general terms, we expect the mechanism discussed here to be active whenever DM predominantly acquires its mass in a phase transition occurring after a significant period of super-cooling.

\section{General mechanism}

We consider extensions of the SM that provide a DM candidate and where all particles get mass from the vacuum expectation value of a scalar $s$, sometimes called 'dilaton'. In the standard freeze-out scenario, DM with mass $M_{\mathrm{DM}}$ would decouple at a temperature $T_{\mathrm{dec}}$, equal to $T_{\mathrm{dec}} \approx M_{\mathrm{DM}} / 25$ if freeze-out reproduces the cosmological DM density. 


\section{$2.1 \quad$ Super-cooling}

In dimension-less models, due to the absence of quadratic terms and vacuum stability (i.e. positive scalar quartics), thermal effects select, as the Universe cools down, the false vacuum where all scalars (in particular the dilaton $s$ and the Higgs $h$ ) have vanishing vacuum expectation values. Around this vacuum, all particles are massless, including DM. The energy density of the Universe receives two contributions: from radiation, $\rho_{\text {rad }}(T)=g_{*} \pi^{2} T^{4} / 30$, and from the vacuum energy of the false vacuum $V_{\Lambda}>0$. We assume that the true vacuum has a nearly zero vacuum energy, as demanded by the observed small cosmological constant.

While the Universe cools, the vacuum energy starts dominating over radiation at some temperature $T=T_{\text {infl }}$ starting a phase of thermal inflation with Hubble constant $H$, such that $V_{\Lambda}$ determines $T_{\text {infl }}$ and $H$ as

$$
\frac{g_{*} \pi^{2} T_{\mathrm{inf}}^{4}}{30}=V_{\Lambda}=\frac{3 H^{2} M_{\mathrm{Pl}}^{2}}{8 \pi}
$$

where $g_{*}$ is the number of relativistic degrees of freedom just before inflation starts. We denote as $a_{\text {inf }}$ the scale factor of the Universe at this stage and write $\mathcal{O}(1)$ factors in Boltzmann approximation (correct within $\pm 10 \%$ ) given that DM might be bosonic or fermionic.

During this phase DM is massless and thereby remains coupled, rather than undergoing thermal freeze-out at $T \sim T_{\text {dec }}$ as e.g. in [17]. All particles, including DM, undergo supercooling: the scale factor of the Universe grows as $a=a_{\text {infl }} e^{H t}$, and the temperature drops as $T=T_{\text {infl }} a_{\text {infl }} / a$.

Super-cooling ends at some temperature $T_{\text {end }}$ with a phase transition towards the true vacuum at $\langle s\rangle,\langle h\rangle \neq 0$ - at the end of this section we will discuss how this happens in the models of interest. During thermal inflation, the scale factor of the Universe inflates by a factor $e^{N}=T_{\text {infl }} / T_{\text {end }}$.

\section{$2.2 \quad$ Reheating}

After the first-order phase transition to the true vacuum, the various particles, including DM, become massive, and the energy density $V_{\Lambda}$ stored in the scalars is transferred to particles, reheating the Universe. If the energy transfer rate $\Gamma$ is much faster than the Hubble rate $H$, the reheating temperature is $g_{\mathrm{RH}}^{1 / 4} T_{\mathrm{RH}}=g_{*}^{1 / 4} T_{\mathrm{infl}}$, where $g_{\mathrm{RH}}$ is the number of reheated degrees of freedom. Otherwise the scalars, before decaying, undergo a period of oscillations and the reheating temperature is lower. During this period, the scalars dilute as matter. When scalars finally decay, their remaining energy density $\rho_{\text {sca }}$ becomes radiation with reheating temperature

$$
T_{\mathrm{RH}} \approx T_{\mathrm{infl}} \min (1, \Gamma / H)^{1 / 2} .
$$

The final DM abundance $Y_{\mathrm{DM}}=n_{\mathrm{DM}} / s$ (where $s=2 \pi^{2} g_{*} T^{3} / 45$ is the entropy density) receives two contributions:

$$
\left.Y_{\mathrm{DM}} \approx Y_{\mathrm{DM}}\right|_{\text {super-cool }}+\left.Y_{\mathrm{DM}}\right|_{\text {sub-thermal }}
$$


The super-cool contribution to the DM abundance is what remains of the original population of formerly massless DM in thermal equilibrium, suppressed by the dilution due to thermal inflation

$$
\left.Y_{\mathrm{DM}}\right|_{\text {super-cool }}=Y_{\mathrm{DM}}^{\mathrm{eq}} \frac{T_{\mathrm{RH}}}{T_{\mathrm{infl}}}\left(\frac{T_{\mathrm{end}}}{T_{\mathrm{inf}}}\right)^{3}, \quad Y_{\mathrm{DM}}^{\mathrm{eq}}=\frac{45 g_{\mathrm{DM}}}{2 \pi^{4} g_{*}} .
$$

The factor $T_{\mathrm{RH}} / T_{\mathrm{infl}}$ arises taking into account that the energy stored in the oscillating inflaton dilutes as matter (rather than as radiation) between the end of inflation and reheating, when it is finally converted to radiation.

The second contribution is the population of DM particles which can be produced from the thermal bath, through scattering effects, after reheating. ${ }^{1}$ If in this way DM has the time to thermalize again, i.e. if $T_{\mathrm{RH}} \gtrsim T_{\mathrm{dec}}$, the supercooled population is erased and this second population reaches thermal equilibrium. In this case DM undergoes a usual freezeout, leading to a relic density independent of whether there was previously a supercooling period. If instead $T_{\mathrm{RH}} \lesssim T_{\mathrm{dec}}$ the super-cool population remains basically unchanged, and the second population is produced with a sub-thermal abundance. This population is determined by the usual Boltzmann equation for $Y_{\mathrm{DM}}=n_{\mathrm{DM}} / s$ in a radiation-dominated Universe, the same equation as the one that controls DM freeze-out. In non-relativistic approximation

$$
\frac{d Y_{\mathrm{DM}}}{d z}=\frac{\lambda}{z^{2}}\left(Y_{\mathrm{DM}}^{2}-Y_{\mathrm{DM}}^{2 \mathrm{eq}}\right), \quad \text { where } \quad z=\frac{M_{\mathrm{DM}}}{T} .
$$

where $\lambda=M_{\mathrm{Pl}} M_{\mathrm{DM}}\left\langle\sigma_{\mathrm{ann}} v_{\mathrm{rel}}\right\rangle \sqrt{\pi g_{\mathrm{SM}} / 45}$ if $\mathrm{DM}$ annihilations have a cross section $\sigma_{\mathrm{ann}}$ dominated by $s$-wave scattering. For $\lambda \gg 1$ freeze-out occurs at $T_{\mathrm{dec}} \approx M_{\mathrm{DM}} / \ln \lambda$; otherwise $\mathrm{DM}$ never thermalizes again after reheating and the term proportional to $Y_{\mathrm{DM}}^{2 \mathrm{eq}}$ can be neglected. Integrating eq. (2.5) the regenerated DM population (starting from $T=T_{\mathrm{RH}}$ ) is

$$
\left.Y_{\mathrm{DM}}\right|_{\text {sub-thermal }}=\lambda \int_{z_{\mathrm{RH}}}^{\infty} \frac{d z}{z^{2}} Y_{\mathrm{eq}}^{2}=\lambda \frac{2025 g_{\mathrm{DM}}^{2}}{128 \pi^{7} g_{\mathrm{SM}}^{2}} e^{-2 z_{\mathrm{RH}}}\left(1+2 z_{\mathrm{RH}}\right) .
$$

Summarising, the super-cool contribution dominates provided that $T_{\mathrm{RH}} \ll T_{\mathrm{dec}}$. If DM interaction are small enough that it does not undergo kinetic recoupling, DM remains colder than in the freeze-out scenario: this has little observational implications [19]; the fact that super-cool DM forms smaller structures could give enhanced tidal fluctuations possibly observable along the lines of [20]. The super-cool population of eq. (2.4) does not depend on DM properties (provided that initially, before supercooling, it was in thermal equilibrium), but only on the amount of super-cooling. As we will see below, eq. (2.4) matches the desired DM abundance provided that $T_{\text {end }}$ is a few orders of magnitude below $T_{\mathrm{RH}}$. If instead $T_{\mathrm{RH}} \lesssim T_{\mathrm{dec}}$, an additional non-thermal DM population is generated after reheating which can also lead to the desired relic density.

\footnotetext{
${ }^{1}$ Production during pre-heating was discussed in [15]. Production during bubble collisions was discussed in $[18]$.
} 


\subsection{The phase transition to the massive vacuum}

Super-cooling ends anyway at the nucleation temperature $T_{\text {nuc }}$ when the rate of thermal vacuum decay becomes faster than the Hubble rate. So far nothing connects the DM mass to the weak scale. However, if this nucleation temperature lies below the QCD phase transition, super-cooling in fact ends sooner, at this transition [21, 22]. The temperature at which super-cooling ends, $T_{\text {end }}$ is approximated by

$$
T_{\text {end }}=\max \left[T_{\text {nuc }}, \min \left(T_{\mathrm{cr}}^{\mathrm{QCD}}, T_{\mathrm{end}}^{\mathrm{QCD}}\right)\right] \text {. }
$$

Indeed, quark condensates form at $T_{\mathrm{cr}}^{\mathrm{QCD}} \sim \Lambda_{\mathrm{QCD}}$. In view of its Yukawa couplings to quarks (in particular to the top) the Higgs acquires a vacuum expectation value $\langle h\rangle_{\mathrm{QCD}} \sim \Lambda_{\mathrm{QCD}}$, which induces a squared mass term $M_{s}^{2}$ for the $s$ scalar. If $M_{s}^{2}$ is negative and bigger in modulus than the thermal $s$ mass (which dominates the potential around the origin in dimension-less theories) $s$ immediately starts rolling down, ending super-cooling at $T_{\mathrm{cr}}^{\mathrm{QCD}}$. Otherwise, $s$ starts rolling at a lower temperature $T_{\mathrm{end}}^{\mathrm{QCD}}$, as soon as its thermal mass becomes smaller than $\left|M_{s}\right|$. When super-cooling is stopped at $T_{\text {end }} \sim \Lambda_{\mathrm{QCD}}$ the amount of super-cooling that reproduces the observed DM abundance is obtained for a DM mass fixed by $T_{\text {end }} \sim \Lambda_{\mathrm{QCD}}$, leading to $T_{\text {end }} / T_{\text {infl }} \sim 10^{-3-4}$, which corresponds to $\mathrm{TeV}$-scale DM. In this way the DM mass gets connected to approximately the weak scale.

\subsection{The baryon asymmetry}

The baryon asymmetry is washed out by the super-cooling factor $e^{3 N}$. Thereby the scenario needs to be supplemented by some mechanism that regenerates the baryon asymmetry around the weak scale, after super-cooling. This can happen, provided that the three Sakharov conditions for baryogenesis are satisfied. ${ }^{2}$

1. First, deviation from thermal equilibrium can be automatically provided by the end of super-cooling, either through a first-order phase transition or through the QCDinduced tachionic instability of $s, h$.

2. Second, violation of baryon number is automatically provided by sphalerons.

3. Third, CP violation.

The contribution from the CKM phase is too small. One possibility is to add an axion $a$ coupled to gluons as $\alpha_{3} a G_{\mu \nu}^{a} \tilde{G}_{\mu \nu}^{a} / 8 \pi f_{a}$ : the observed baryon asymmetry can be obtained for values of the axion decay constant allowed by data, $f_{a} \sim 10^{11} \mathrm{GeV}[25]$. However, depending on the axion model, such a large scale risks conflicting with our assumption of a dimensionless theory where the weak scale is dynamically-generated [3]. It would be nice if one could generate a large effective $f_{a}$ from weak-scale loops, as attempted in [26]. It seems easier to devise scale-invariant models with extended interactions introduced ad hoc to violate CP: either extra Yukawa couplings or extra scalar quartics. One possibility is adding a second Higgs doublet such that the scalar potential contains one CP-violating phase that can lead

\footnotetext{
${ }^{2}$ See $[23,24]$ for recent discussions.
} 
to baryogengesis [27]. However this also contributes to electric dipoles, and flavour data agree with the SM with one Higgs doublet. Furthermore, detailed computations are needed to establish if the phase transition predicted by the model is enough out of equilibrium.

In the absence of a lepton asymmetry, in the above context the reheating temperature must remain below the decoupling temperature of electroweak sphalerons, $T_{\mathrm{sph}} \approx 132 \mathrm{GeV}$, otherwise sphalerons reach thermal equilibrium and wash-out the baryon asymmetry.

Given that observed neutrino masses anyhow demand an extension of the Standard Model, an appealing alternative possibility developed in the following is low-scale leptogenesis, where new neutrino physics generates a lepton asymmetry, converted by sphalerons into the desired baryon asymmetry.

\section{Model with $\mathrm{SU}(2)_{X}$ gauge group}

The considerations above are fully relevant for basically any dimensionless model that contains a DM candidate (see e.g. [28-55]). Here we consider the model of [10] where the SM gauge group is extended adding an extra $\mathrm{SU}(2)_{X}$ with gauge coupling $g_{X}$, and the field content is extended adding a scalar $S$, doublet under the extra $\mathrm{SU}(2)_{X}$, neutral under the SM gauge group. The Yukawa interactions are those of the SM. The theory is assumed to be dimension-less, such that the tree-level scalar potential is

$$
V=\lambda_{H}|H|^{4}-\lambda_{H S}|H S|^{2}+\lambda_{S}|S|^{4} .
$$

This model generates the weak scale through Coleman-Weinberg dynamical symmetry breaking: the scalar doublets acquire vacuum expectation values and can be written as

$$
S=\frac{1}{\sqrt{2}}\left(\begin{array}{l}
0 \\
s
\end{array}\right), \quad H=\frac{1}{\sqrt{2}}\left(\begin{array}{l}
0 \\
h
\end{array}\right)
$$

without loss of generality. The Coleman-Weinberg mechanism takes place because the quartic $\lambda_{S}$ runs as

$$
\beta_{\lambda_{S}} \equiv \frac{d \lambda_{S}}{d \ln \mu}=\frac{1}{(4 \pi)^{2}}\left[\frac{9 g_{X}^{4}}{8}-9 g_{X}^{2} \lambda_{S}+2 \lambda_{H S}^{2}+24 \lambda_{S}^{2}\right] \approx \frac{1}{(4 \pi)^{2}} \frac{9 g_{X}^{4}}{8}
$$

becoming negative at low energy below some scale $s_{*}$, such that the one-loop potential is approximated as

$$
V_{1}(s) \approx \beta_{\lambda_{S}} \frac{s^{4}}{4} \ln \frac{s}{s_{*}}
$$

which has a minimum at $\langle s\rangle=w=s_{*} e^{-1 / 4}$. The $\mathrm{SU}(2)_{X}$ vectors acquire a mass $M_{X}=g_{X} w / 2$, and are stable DM candidates. Thereby DM has $g_{\mathrm{DM}}=9$ degrees of freedom, including the components of $S$ 'eaten' by the massive vectors in the broken phase. The model has only two free parameters beyond the ones of the SM: we will use $g_{X}$ and $M_{X}$ as free parameters.

We compute the other masses assuming, for simplicity, that $\lambda_{H S}$ is positive and small. Then $\langle s\rangle=w$ induces a Higgs vev $\langle h\rangle=v$ equal to $v / w=\sqrt{\lambda_{H S} / 2 \lambda_{H}}$, where $\lambda_{H} \approx 0.126$ 
is the SM Higgs quartic, up to small corrections. This fixes the value of $\lambda_{H S}$ needed to reproduce the desired EW vacuum. The $s$ mass is $M_{s}=w \sqrt{\beta_{\lambda_{S}}}$. Assuming $M_{s} \ll M_{h}$, the $s / h$ mixing angle is $\alpha \simeq-v / w$. Finally, a dimension-full constant $V_{\Lambda} \approx \beta_{\lambda_{S}} w^{4} / 16 \approx$ $9 M_{X}^{4} / 8(4 \pi)^{2}$ must be added to the potential such that the true vacuum at $s=w$ has zero energy. This is the usual tuning of the cosmological constant.

\subsection{Super-cooling}

At finite temperature the potential receives thermal corrections $V_{T}$, dominated by

$$
V_{T}(s)=\frac{9 T^{4}}{2 \pi^{2}} f\left(\frac{M_{X}}{T}\right)+\frac{T}{4 \pi}\left[M_{X}^{3}-\left(M_{X}^{2}+\Pi_{X}\right)^{3 / 2}\right]
$$

where $f(r)=\int_{0}^{\infty} x^{2} \ln \left(1-e^{-\sqrt{x^{2}+r^{2}}}\right) d x$ and $\Pi_{X}=11 g_{X}^{2} T^{2} / 6$ is the thermal propagator for the longitudinal $X$ component which accounts for re-summation of higher order ringdiagrams $[29,56]$. At small field values, the potential is approximated by positive thermal masses for the scalars $s$ and $h$

$$
M_{s}^{2 T}=\frac{3}{16} g_{X}^{2} T^{2}, \quad M_{h}^{2 T}=\left(\frac{3}{16} g_{2}^{2}+\frac{1}{16} g_{Y}^{2}+\frac{1}{4} y_{t}^{2}+\frac{1}{2} \lambda_{H}\right) T^{2},
$$

such that the thermal vacuum is $\langle s\rangle=\langle h\rangle=0$. As the Universe cools down, a deeper true vacuum appears below a critical temperature $T_{\text {cr }}$, equal to $0.31 M_{X}$ if $g_{X} \lesssim 0.7 \mathrm{such}$ that ring diagrams can be neglected, and roughly a factor $g_{X} / 0.7$ larger otherwise. Given that dimension-less theories only have thermal masses and quartics, the Universe remains trapped in the false vacuum at $s=h=0$ down to some temperature $T_{\text {end }}$.

Thermal inflation begins at the temperature $T_{\text {infl }}$ at which the vacuum energy starts dominating with respect to radiation. Applying eq. (2.1) to our model gives

$$
T_{\mathrm{infl}}=\left(\frac{135}{64 g_{*}}\right)^{1 / 4} \frac{M_{X}}{\pi} \approx \frac{M_{X}}{8.5}, \quad H=\sqrt{\frac{3}{\pi}} \frac{M_{X}^{2}}{4 M_{\mathrm{Pl}}} .
$$

\subsection{End of super-cooling}

During super-cooling, $s$ and $h$ are kept to 0 by thermal masses. The temperature $T_{\text {end }}$ at which thermal inflation ends has the form anticipated in eq. (2.7). First, we consider the possibility that thermal inflation ends through nucleation and compute $T_{\text {nuc }}$. We solve numerically the bounce equation ${ }^{3}$

$$
s^{\prime \prime}(r)+\frac{2}{r} s^{\prime}(r)=\frac{d V}{d s}, \quad s^{\prime}(0)=0, \quad \lim _{r \rightarrow \infty} s(r)=0
$$

and use it to calculate the thermal bounce action

$$
\frac{S_{3}(T)}{T}=\frac{4 \pi}{T} \int d r r^{2}\left[\frac{1}{2} s^{\prime}(r)^{2}+V(s(r))\right] .
$$

\footnotetext{
${ }^{3}$ We assume that the thermal bounce is time-independent and $\mathrm{O}(3)$-symmetric, although this might fail for dimension-less potentials [57].
} 

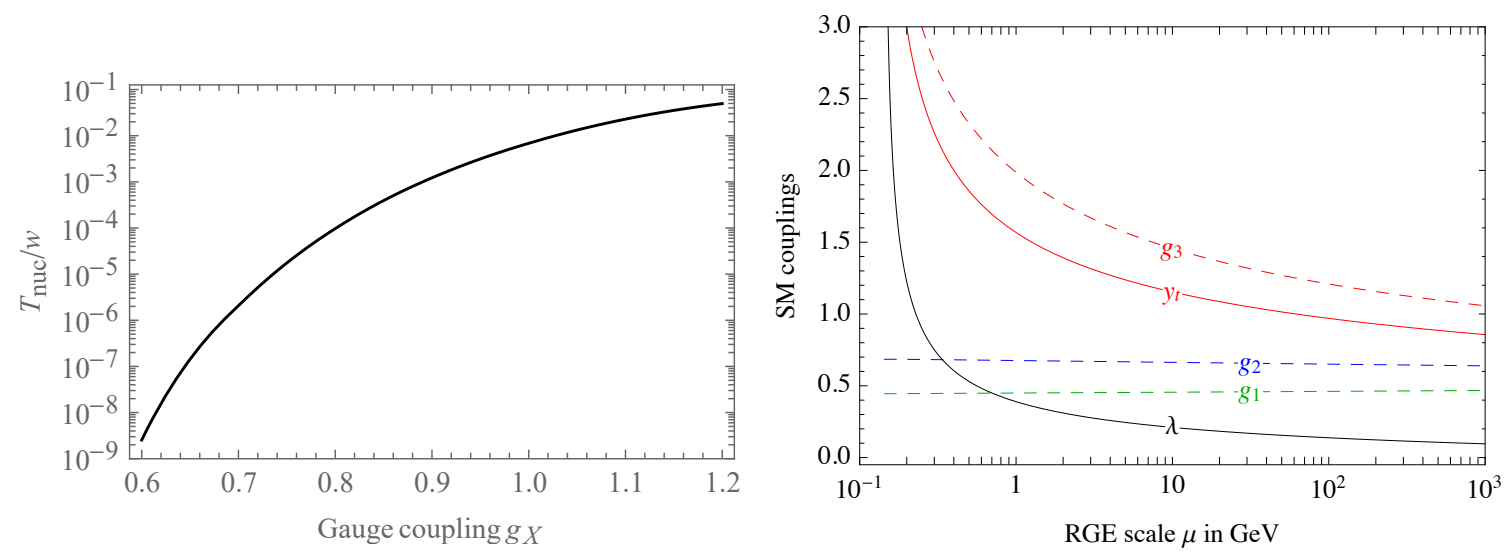

Figure 1. Left: the nucleation temperature given by vacuum decay, ignoring the QCD phase transition. Right: 3-loop RGE running in the massless SM.

At $T \ll w$ the potential can be approximated as $\frac{1}{2} M_{s}^{2 T} s^{2}+\frac{1}{4} \lambda_{S}(T) s^{4}$ where $\lambda(T)<0$ is the quartic coupling renormalized at $T$, and the bounce action as $S_{3} / T \approx 6.0 \pi M_{s}^{T} / T\left|\lambda_{S}\right| \approx$ $8.2 g_{X} /\left|\lambda_{S}\right|[21]$. Nucleation happens at the temperature $T_{\text {nuc }}$ where the tunnelling rate is comparable to the Hubble rate, $S_{3}\left(T_{\text {nuc }}\right) / T_{\text {nuc }} \approx 4 \ln M_{\mathrm{Pl}} / M_{X} \approx 142$. The numerical results are shown in figure 1: $T_{\text {nuc }}$ is very small for small $g_{X}$.

In such a case, QCD stops super-cooling earlier [21, 22]. In the ordinary QCD chiral phase transition scenario where quarks are massive, this phase transition happens at $T_{\mathrm{cr}}^{\mathrm{QCD}} \approx 154 \pm 9 \mathrm{MeV}$ [58]. However, during super-cooling all quarks are massless, which leads to a smaller value of $\alpha_{3}$ at low energy. Figure 1 shows the running of the SM couplings: $\alpha_{3}(\bar{\mu})$ diverges at $\Lambda_{\mathrm{QCD}}^{h=0} \approx 144 \mathrm{MeV}$, with $\Lambda_{\overline{\mathrm{MS}}}^{(6)}=(89 \pm 7) \mathrm{MeV}$ if only $\alpha_{3}$ is kept in the RGE [59]. Then the QCD chiral phase transition happens at a lower temperature, $T_{\mathrm{cr}}^{\mathrm{QCD}} \sim 85 \mathrm{MeV}$ according to the estimate of [22, 60]. When a zero-mode quark condensate forms, the Yukawa coupling $y_{t} h\left\langle t_{L} t_{R}\right\rangle / \sqrt{2}+$ h.c. induces a linear term in the Higgs potential, such that the Higgs acquires a $T$-dependent vacuum expectation value $\langle h\rangle_{\mathrm{QCD}}$. Given that the couplings $y_{t}$ and $\lambda_{H}$ too run to non-perturbative values (see figure 1 ) $\langle h\rangle_{\mathrm{QCD}}$ can at best be estimated. We will proceed assuming $\langle h\rangle_{\mathrm{QCD}} \approx 100 \mathrm{MeV}$, up to order one factors.

Next, $\langle h\rangle$ induces a mass term for the $s$ scalar, $M_{s}^{2}=-\lambda_{H S}\langle h\rangle^{2} / 2$. If $\lambda_{H S}<0$, the positive $M_{s}^{2}$ delays the end of thermal inflation. If $\lambda_{H S}$ is positive (as needed to break $\mathrm{SU}(2)_{L}$ at the true minimum) the negative $M_{s}^{2}$ triggers the end of thermal inflation: $s$ too starts rolling down as soon as its extra mass term $M_{s}$ becomes larger than its thermal mass $M_{s}^{T}$ in eq. (3.6). If $\lambda_{H S}$ is large enough, this happens immediately at $T_{\text {end }}=T_{\mathrm{cr}}^{\mathrm{QCD}}$; otherwise this happens later at a lower temperature

$$
T_{\mathrm{end}}^{\mathrm{QCD}}=\sqrt{\frac{8 \lambda_{H S}}{3}} \frac{\langle h\rangle_{\mathrm{QCD}}}{g_{X}} \approx \frac{0.1\langle h\rangle_{\mathrm{QCD}}}{M_{X} / \mathrm{TeV}} .
$$

In the present model the thermal mass $M_{s}^{T}$ is dominated by DM vectors, so that thermal inflation ends when their density is diluted enough.

We now have all the factors that determine $T_{\text {end }}$ in eq. (2.7). During super-cooling, the Universe inflates by a factor $T_{\text {infl }} / T_{\text {end }}$ plotted in figure $2 \mathrm{a}$. The horizontal part of the 
Number of $e$-folds $N$

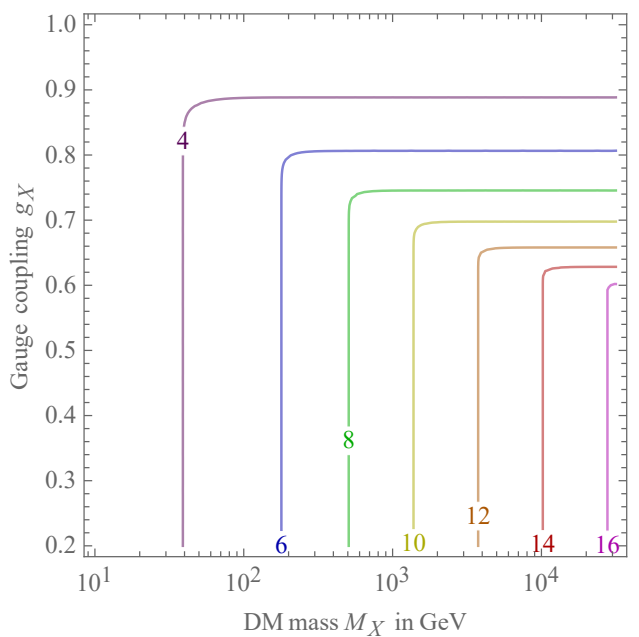

$T_{\mathrm{RH}}$ and $M_{s}$ in $\mathrm{GeV}$

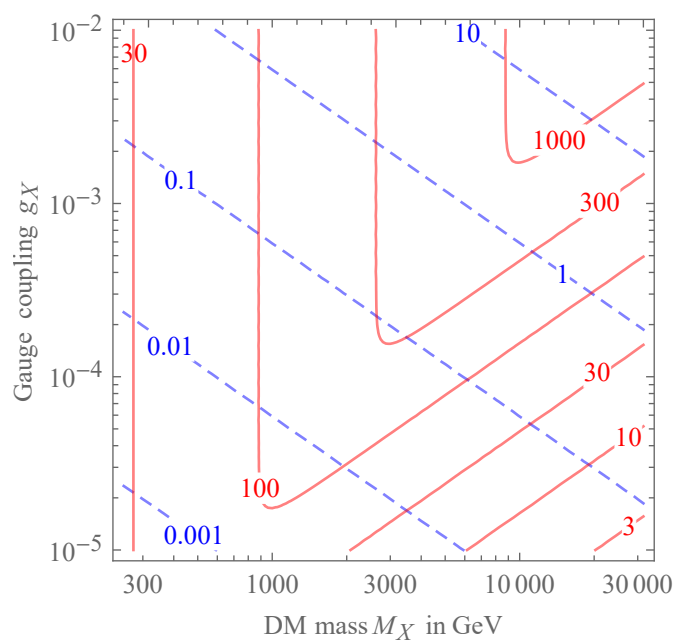

Figure 2. Left: number of $e$-folds of thermal inflation, $N=\ln T_{\text {infl }} / T_{\text {end }}$ for $\langle h\rangle_{\mathrm{QCD}}=100 \mathrm{MeV}$. Right: reheating temperature in $\mathrm{GeV}$ (solid red curves) and $M_{s} / \mathrm{GeV}$ (diagonal dashed lines).

contours corresponds to end of super-cooling via vacuum decay, and the vertical part to the QCD-triggered end.

\subsection{Reheating}

After the end of inflation, the scalars oscillate around the true minimum, dissipating their energy density $\rho_{\text {sca }}$ with some rate $\Gamma$ into radiation that acquires energy density $\rho_{\text {rad }}=g_{*} \pi^{2} T^{4} / 30$. The rolling fields $s$ and $h$ finally settle at the true minimum. The scale factor $a$ and the various components evolve as

$$
\left\{\begin{aligned}
\frac{\dot{a}}{a} & =H=\frac{1}{\bar{M}_{\mathrm{Pl}}} \sqrt{\frac{\rho_{\mathrm{sca}}+\rho_{\mathrm{rad}}}{3}} \\
\dot{\rho}_{\mathrm{sca}} & =-(3 H+\Gamma) \rho_{\mathrm{sca}} \\
\dot{\rho}_{\mathrm{rad}} & =-4 H \rho_{\mathrm{rad}}+\Gamma \rho_{\mathrm{sca}} \\
\dot{n}_{\mathrm{DM}} & =-3 H n_{\mathrm{DM}}+\langle\sigma v\rangle_{\mathrm{ann}}\left(n_{\mathrm{DM}}^{\mathrm{eq} 2}-n_{\mathrm{DM}}^{2}\right)+\langle\sigma v\rangle_{\mathrm{semi}} n_{\mathrm{DM}}\left(n_{\mathrm{DM}}^{\mathrm{eq}}-n_{\mathrm{DM}}\right) .
\end{aligned}\right.
$$

Thereby $\rho_{\text {sca }}(t)=\rho_{\text {sca }}\left(t_{\text {end }}\right) e^{-\Gamma\left(t-t_{\text {end }}\right)}\left[a\left(t_{\text {end }}\right) / a(t)\right]^{3}$. This roughly means that the inflaton $s$ reheats the Universe up to the temperature

$$
T_{\mathrm{RH}}=\left(\frac{45}{4 \pi^{3} g_{*}}\right)^{1 / 4} M_{\mathrm{Pl}}^{1 / 2} \min (H, \Gamma)^{1 / 2}=T_{\text {infl }} \min \left(1, \frac{\Gamma}{H}\right)^{1 / 2} .
$$

We need to compute $\Gamma$. The scalar equations of motion are

$$
\left\{\begin{array}{rl}
\ddot{s}+\left(3 H+\Gamma_{s}\right) \dot{s} & =-\partial V / \partial s \\
\ddot{h}+\left(3 H+\Gamma_{h}\right) \dot{h} & =-\partial V / \partial h
\end{array} \quad \Rightarrow \quad \frac{d}{d t}(K+V)=-6 H K-\Gamma_{h} K_{h}-\Gamma_{s} K_{s}\right.
$$

where $K=K_{h}+K_{s}=\left(\dot{s}^{2}+\dot{h}^{2}\right) / 2$ is the scalar kinetic energy. Given that scalar masses are much bigger than $H$, averaging over the fast oscillations around the minimum, one finds 
that the scalar energy $\rho_{\text {sca }}=\langle K+V\rangle=2\langle K\rangle$ red-shifts as non relativistic matter, in the limit $\Gamma_{h}=\Gamma_{s}=0$.

All masses stay positive around the true minimum, so decays are not enhanced by parametric resonances. Most of the energy is stored in $s$, but its decay rate $\Gamma_{s}$ can be smaller than $H$. On the other hand the Higgs potential energy is sub-leading, while its decay rate $\Gamma_{h} \approx 4 \mathrm{MeV}$ is fast. Thereby the decay rate $\Gamma$ of the combined system is controlled by the rate for energy transfer from $s$ to $h$ due to the $\lambda_{H S}$ interaction. We compute $\Gamma$ by solving the equations of motion in linear approximation around the minimum

$$
\begin{aligned}
\ddot{h}+\left(3 H+\Gamma_{h}\right) \dot{h} & =-\tilde{M}_{h}^{2}(h-v)+\lambda_{H S} v w(s-w) \\
\ddot{s}+\left(3 H+\Gamma_{s}\right) \dot{s} & =-\tilde{M}_{s}^{2}(s-w)+\lambda_{H S} v w(h-v),
\end{aligned}
$$

where $\tilde{M}_{h}^{2}=2 \lambda_{H} v^{2}, \tilde{M}_{s}^{2}=\beta_{\lambda_{S}} w^{2}$. The complex frequencies of the normal modes of damped oscillations in the limit $\Gamma_{s}, H \ll \Gamma_{h} \ll M_{h}$ are $\omega \simeq M_{h} \pm i\left(3 H+\Gamma_{h}\right) / 2$ and $\omega \simeq M_{s} \pm i(3 H+\Gamma) / 2$ with

$$
\Gamma=\Gamma_{h} \sin ^{2} \alpha+\Gamma_{s} \cos ^{2} \alpha
$$

where $\alpha \simeq-v / w=\sqrt{\lambda_{H S} / 2 \lambda_{H}} \ll 1$ is the angle that diagonalizes the mass matrix.

Figure $2 \mathrm{~b}$ shows the numerical results for $T_{\mathrm{RH}}$. For a given $M_{X}$, reheating is instantaneous provided that $g_{X}$ is large enough, so that $T_{\mathrm{RH}}=T_{\mathrm{infl}} \simeq M_{X} / 8.5$, corresponding to vertical contour lines in figure $2 \mathrm{~b}$. For smaller $g_{X}$ the reheating temperature is suppressed. ${ }^{4}$

\subsection{The dark matter abundance}

The DM candidates are the $\mathrm{SU}(2)_{X}$ vectors with mass $M_{X}$. In the usual scenario where they are thermal relics, the observed DM abundance is reproduced for $g_{X}^{2} \approx M_{X} / \mathrm{TeV}[10]$ and super-cooling is negligible. Smaller values of $g_{X}$ lead to super-cooling, realising the novel DM production mechanism proposed here: the DM abundance is the sum of the super-cool population, plus the sub-thermal population, eq. (2.3).

We compute the super-cool DM population specializing eq. (1.1) and eq. (2.4) to the present model. We find that the super-cool abundance reproduces the observed DM abundance when the end of super-cooling is triggered by the QCD phase transition as $T_{\text {end }}=T_{\text {end }}^{\mathrm{QCD}}$ (eq. (3.10)), and when reheating is instantaneous. Thereby $T_{\mathrm{RH}}=T_{\mathrm{infl}} \approx M_{X} / 8.5$ and the $\mathrm{DM}$ abundance simplifies to

$$
\left.\Omega_{\mathrm{DM}} h^{2}\right|_{\text {super-cool }} \approx 3.7 \times 10^{-3}\left(\frac{\langle h\rangle_{\mathrm{QCD}}}{100 \mathrm{MeV}}\right)^{3}\left(\frac{\mathrm{TeV}}{M_{X}}\right)^{5} .
$$

It does not depend on $g_{X}$, giving rise to the vertical contours in the $\left(M_{X}, g_{X}\right)$ plane in the left panel of figure 3 .

As anticipated, this is not the end of the story: one needs to take into account the effects of thermal scatterings after reheating. One needs to evolve the Boltzmann equation

\footnotetext{
${ }^{4}$ For very small $g_{X}$ one can have $T_{\mathrm{RH}} \lesssim 100 \mathrm{MeV}$ so that macroscopic six-flavour quark nuggets [63] could contribute to the DM relic density.
} 

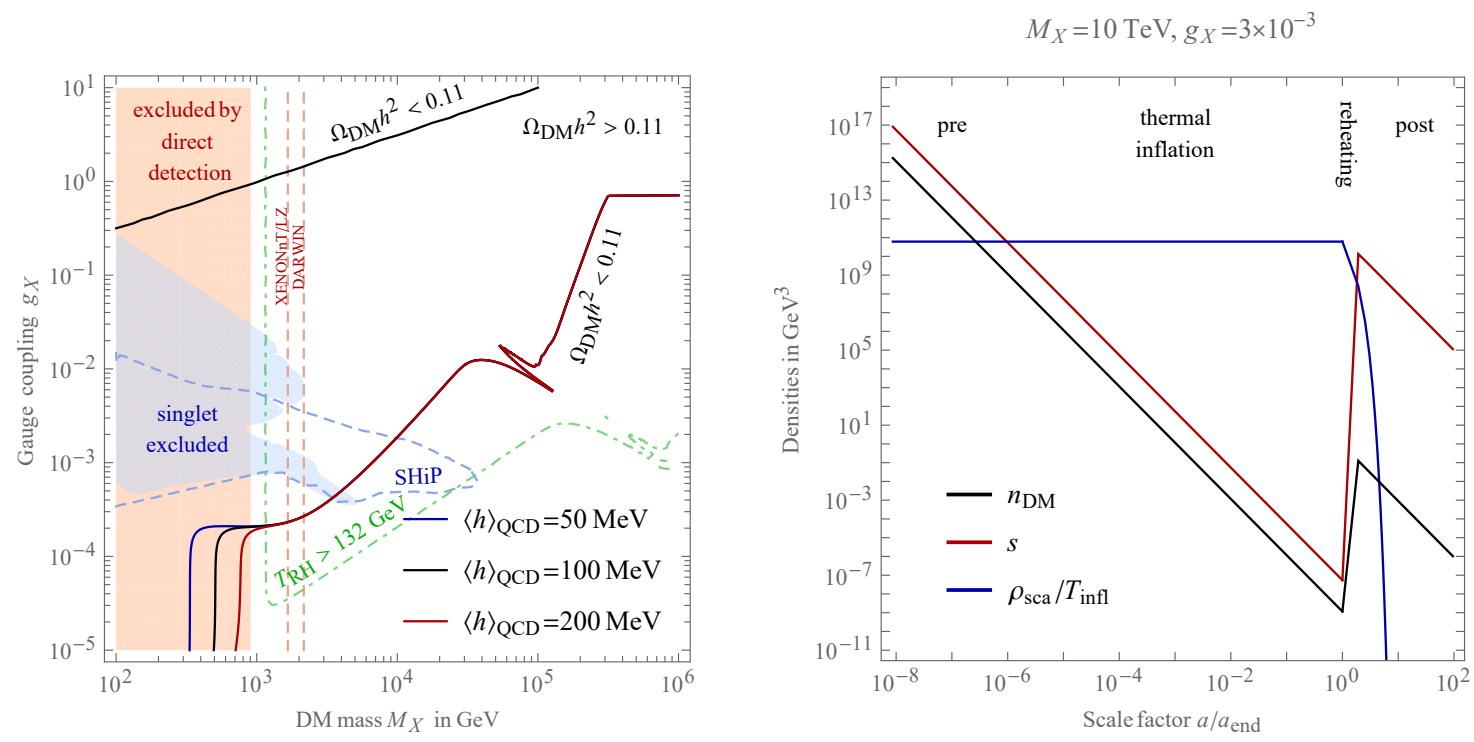

Figure 3. Left:The observed DM abundance is reproduced along the solid curves, computed for different values of the uncertain QCD factor $\langle h\rangle_{\mathrm{QCD}}$. The region shaded in orange (blue) is excluded by direct DM searches (collider searches for the singlet $s$ ). Dashed curves show future detection prospects. Right: sample evolution of the DM density, of entropy, of the scalar energy.

in eq. (3.11) starting from the initial condition $Y_{\mathrm{DM}}\left(T_{\mathrm{RH}}\right)=\left.Y_{\mathrm{DM}}\right|_{\text {super-cool }}$. The $s$-wave cross-sections for DM annihilations $V V \leftrightarrow s s$ and semi-annihilations $V V \leftrightarrow V s$ are [10]

$$
\langle\sigma v\rangle_{\mathrm{ann}}=\frac{11 g_{X}^{4}}{6912 \pi M_{X}^{2}}, \quad\langle\sigma v\rangle_{\mathrm{semi}-\mathrm{ann}}=\frac{g_{X}^{4}}{128 \pi M_{X}^{2}} .
$$

In the extreme case where the reheating temperature is larger than the DM decoupling temperature, the super-cool population is erased and substituted by the usual thermal relic population. Otherwise, the super-cool population is negligibly suppressed, and complemented by the additional sub-thermal population of eq. (2.6). For instantaneous reheating $\left(z_{\mathrm{RH}} \approx 8.4\right)$ this evaluates to $\left.\Omega_{\mathrm{DM}} h^{2}\right|_{\text {sub-thermal }} \approx 0.110\left(g_{X} / 0.00020\right)^{4}$, giving rise to the horizontal part of the contour at $g_{X} \approx 10^{-4}$ in the $\left(M_{X}, g_{X}\right)$ plane of figure 3 . At larger $M_{X}$ reheating is no longer instantaneous, giving rise to the oblique part of the contour in figure 3a, which shows the complete numerical results for the DM density. Finally, for even larger masses $M_{X} \gtrsim 300 \mathrm{TeV}$ the super-cool abundance reproduces the observed DM relic density with $g_{X} \sim O(1)$, so that super-cooling is ended by nucleation, leading to a DM abundance that depends mainly on $g_{X}$ and not on $M_{X}$. Therefore, DM masses can be even $\mathrm{PeV}$-scale or larger, higher than those allowed with freeze-out and perturbative couplings. At $M_{X} \approx 100 \mathrm{TeV}$ one has $M_{s} \simeq M_{h}$, so that a resonance-like feature in the mixing angle $\alpha$, and consequently in $T_{\mathrm{RH}}$ and $\Omega_{\mathrm{DM}}$, appears.

In the region of the parameter space relevant for the present work, the Spin-Independent cross section for DM direct detection is dominantly mediated by $s$ and simplifies to

$$
\sigma_{\mathrm{SI}}=\frac{m_{N}^{4} f^{2}}{16 \pi v^{2}}\left(\frac{1}{m_{s}^{2}}-\frac{1}{m_{h}^{2}}\right)^{2} g_{X}^{2} \sin ^{2} 2 \alpha \simeq \frac{64 \pi^{3} f^{2} m_{N}^{4}}{81 M_{X}^{6}} \approx 0.610^{-45} \mathrm{~cm}^{2}\left(\frac{\mathrm{TeV}}{M_{X}}\right)^{6}
$$


where $f \approx 0.295$ is the nucleon matrix element and $m_{N}$ is the nucleon mass. So $\sigma_{\mathrm{SI}}<1.510^{-45} \mathrm{~cm}^{2}\left(M_{X} / \mathrm{TeV}\right)[64]$ for $M_{X}>0.88 \mathrm{TeV}$.

Figure 3a also shows the existing bounds and future discovery prospects, coming both from searches of dark matter via direct detection, and from collider searches for $s$. The region shaded in orange is excluded by direct searches at XENON1T [64] and the dashed vertical lines denote the future sensitivity of XENONnT [65], LZ [66] and DARWIN [67]. The direct detection cross section is suppressed by two powers of the small $g_{X}$ coupling and enhanced by the exchange of the $s$ scalar state, which is light, see figure $2 \mathrm{~b}$. As a result the direct detection constraint is significant. The region shaded in blue is excluded by collider searches for $s$ : the dominant collider bounds come from $s \rightarrow e e, \mu \mu$ at CHARM and $B \rightarrow K^{*} \mu \mu$ at LHCb, as summarized in [68]. The dashed blue curves indicates the future sensitivity of $\operatorname{SHiP}[68,69]$.

Finally, figure $3 \mathrm{~b}$ shows a sample example of the evolution of the DM density, of entropy, of the energy density in scalars: the latter dominate during super-cooling, while $n_{\mathrm{DM}}$ and $s$ decrease equally. At reheating almost all of this energy is transferred to entropy, and only a small fraction goes to massive DM.

\subsection{The baryon asymmetry}

The reheating temperature $T_{\mathrm{RH}}$ in the $\mathrm{SU}(2)_{X}$ model is shown in figure $2 \mathrm{~b}$ and can be either smaller or larger than $T_{\mathrm{sph}}$, see figure $3 \mathrm{a}$. In the first case cold baryogenesis might be a viable option (with extra CP-violation), while leptogenesis is a clear option in the second case.

Leptogenesis can in particular be achieved if one adds right-handed neutrinos $N$, with Yukawa couplings $Y_{N} N L H$, and an extra real scalar singlet $S^{\prime}$, with quartic potential couplings and a Yukawa coupling $y_{S} S^{\prime} N^{2} / 2$ that breaks lepton number. This induces a mass $M_{N}$ for $N$ if $S^{\prime}$ acquires a vev.

Low-scale leptogenesis with right-handed neutrinos and their Yukawa couplings can then occur either:

1) via resonant CP-violating decays of the right-handed neutrinos [70-72];

2) via oscillations of right-handed neutrinos with total $L$ lepton number conservation (ARS framework $[73,74]$ );

3) via $L$-violating Higgs decays [75, 76].

Barring fine-tunings in the structure of Yukawa couplings, a sizeable lepton asymmetry needs quasi-degenerate right-handed neutrinos, at the per-million level. The timevariation of their masses (while scalars relax to their minimum) relaxes the amount of quasi-degeneracy, a scenario we will not further explore here.

The first possibility, resonant CP-violating decays, mostly produces an asymmetry for $T \sim M_{N}$ and thereby needs $T_{\mathrm{RH}} \gtrsim M_{N}$. Indeed the right-handed neutrino mass must be sizeably above the electroweak scale to allow $N \rightarrow H L$ decays and to produce efficiently the asymmetry before $T_{\mathrm{sph}}$. In the super-cool DM production scenario above, the reheating 


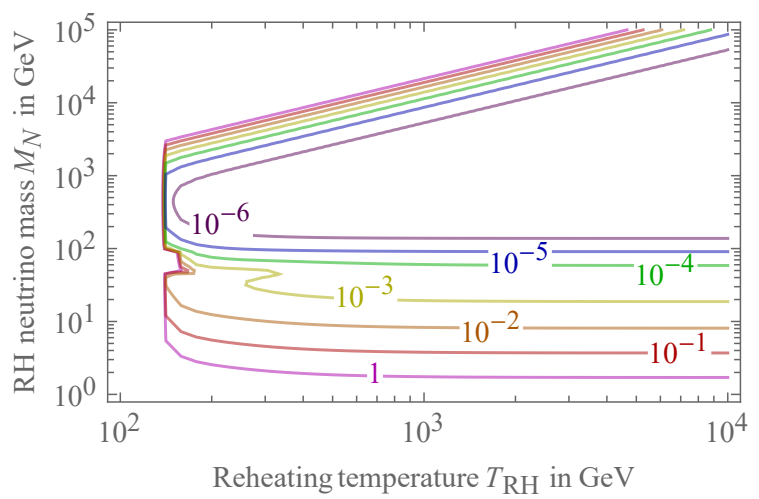

Figure 4. Leptonic CP asymmetry $\epsilon_{\mathrm{CP}}$ needed to obtain successful leptogenesis, assuming righthanded neutrinos in thermal equilibrium at the temperature $T_{\mathrm{RH}}$. For $M_{N} \gtrsim M_{h}\left(M_{N} \lesssim M_{h}\right)$ the asymmetry comes from decays of right-handed neutrinos (of the Higgs), and the needed $\epsilon_{\mathrm{CP}}$ is obtained from right-handed neutrinos degenerate at the $\Delta M_{N} / M_{N} \approx 10^{-7}$ level (at the $10^{-5}$ level). We fixed the Yukawa couplings to $\left|Y_{N}\right|^{2} v^{2} / M_{N}=10^{-11} \mathrm{eV}$.

temperature can be above a $\mathrm{TeV}$, see figure $2 \mathrm{~b}$. This longer period of electroweak symmetry breaking restoration makes this scenario easily viable. This is shown in figure 4. Successful leptogenesis implies a lower bound on the reheating temperature, depending on $M_{N}$, which implies a lower bound on $M_{X}$.

The second possibility of low scale leptogenesis, right-handed neutrino oscillations in $L$-conserving processes, requires lighter $N$, around the $\mathrm{GeV}$ scale. However, it is in general fully operational at temperatures orders of magnitudes larger than the electroweak scale. Thus, except in special situations, in our context it is suppressed by the low reheating temperature.

The third possibility, $L$-violating Higgs decay (which requires right-handed neutrino mass between a $\mathrm{GeV}$ up to the Higgs mass), produces dominantly the baryon asymmetry at temperatures just above the sphaleron decoupling temperature $T_{\mathrm{sph}}$. Therefore, one only needs $T_{\mathrm{RH}} \gtrsim T_{\mathrm{sph}}$, which can be realised in the allowed parameter space of figure 3 . This mechanism explains why in figure 4, which combines the leptogenesis contributions from $L$-violating $N$ and $H$ decays, leptogenesis is viable for masses below the Higgs boson mass and $T_{\mathrm{RH}} \geq T_{\mathrm{sph}}$. We solved Boltzmann equations taken from [75] in the singleflavour approximation for the SM leptons. These do not take into account the reheating temperature suppressed purely-flavoured ARS contribution.

In all cases (including the model discussed in section 4 below), leptogenesis is significantly facilitated by the super-cool mechanism, since the required gauge couplings are small and do not dilute the asymmetry as they do, instead, in the WIMP regime [77, 78], where successful leptogenesis would hardly be possible.

\section{Model with $\mathrm{U}(1)_{B-L}$ gauge group}

We here study a different super-cool DM model. Given that leptogenesis seems the most plausible option for baryogenesis, a natural possibility is gauging $B-L$, such that right- 
handed neutrinos become necessary for anomaly cancellation. This makes $\mathrm{SU}(2)_{X}$ no longer necessary for dynamically breaking scale invariance: the role of $\mathrm{SU}(2)_{X}$ can be played by $\mathrm{U}(1)_{B-L}$, in a similar way. The scalar doublet $S$ of $\mathrm{SU}(2)_{X}$ is replaced by a complex scalar, $S$, charged under $\mathrm{U}(1)_{B-L}$. The gauge coupling $g_{B-L}$ can drive the scalar quartic $\lambda_{S}$ to run negative around the weak scale, such that the scalar $S$ again acquires a vacuum expectation value. As a result, the $B-L$ gauge boson $Z^{\prime}$ acquires a mass, eating the would-be Majoron. The weak symmetry is again broken thanks to a $-\lambda_{H S}|S H|^{2}$ term in the potential with $\lambda_{H S}>0$.

Assuming that it has a $B-L$ charge equal to 2 , the $S$ scalar can be identified with the field that gives mass to right handed neutrinos $N$, through a $Y_{S} S N^{2} / 2$ Yukawa interaction.

A disadvantage of this model (compared to the $\mathrm{SU}(2)_{X}$ model) is that the $Z^{\prime}$ cannot be DM: it decays into SM fermions, as they are charged under $B-L$; furthermore $B-L$ can have a kinetic mixing with hypercharge. We thereby add one extra scalar singlet $\phi$, with no hypercharge and $B-L$ charge $q_{\phi}$ chosen such that it is stable: for simplicity we assume $q_{\phi}=1$. This makes DM absolutely stable due to the fact that $\phi$ is odd under the $\mathbb{Z}_{2} \subset \mathrm{U}(1)_{B-L}$ symmetry, which remains unbroken because $S$ has $B-L$ charge $2 .^{5}$

Summarising, the model is described by gauge-invariant kinetic terms, plus the dimension-less scalar potential plus a constant

$$
V=\lambda_{H}|H|^{4}+\lambda_{S}|S|^{4}+\lambda_{\phi}|\phi|^{4}-\lambda_{H S}|H S|^{2}+\lambda_{S \phi}|S \phi|^{2}+\lambda_{H \phi}|H \phi|^{2}+V_{\Lambda}
$$

plus the Yukawa interactions

$$
\mathscr{L}_{\text {Yuk }}=\mathscr{L}_{\text {Yuk }}^{\mathrm{SM}}+Y_{N} N L H+Y_{S} S \frac{N^{2}}{2}+\text { h.c. }
$$

After symmetry breaking the scalar fields can be written as

$$
S=\frac{s}{\sqrt{2}}, \quad H=\frac{1}{\sqrt{2}}\left(\begin{array}{l}
0 \\
h
\end{array}\right) .
$$

At one loop, the $\lambda_{S}$ quartic runs as

$$
\beta_{\lambda_{S}} \equiv \frac{d \lambda_{S}}{d \ln \mu}=\frac{1}{(4 \pi)^{2}}\left[96 g_{B-L}^{4}-Y_{S}^{4}+2 \lambda_{H S}^{2}+\lambda_{S \phi}^{2}+20 \lambda_{S}^{2}+\lambda_{S}\left(2 Y_{S}^{2}-48 g_{B-L}^{2}\right)\right]
$$

becoming negative at low energy below some scale $s_{*}$, such that its one-loop potential is approximated as

$$
V_{1}(s) \approx \beta_{\lambda_{S}} \frac{s^{4}}{4} \ln \frac{s}{s_{*}}
$$

which develops a minimum at $\langle s\rangle=w=s_{*} e^{-1 / 4}$. This generates

$$
M_{Z^{\prime}}=2 g_{B-L} w, \quad M_{N}=Y_{S} w
$$

\footnotetext{
${ }^{5}$ This $B-L$ gauge group has been considered in its scale invariant version in $[22,79]$ as a model for neutrino masses (without DM) and in [41], with a DM scalar particle which has no $B-L$ charge (in order to avoid direct detection bounds) and is stabilised adding an extra $\mathbb{Z}_{2}$ symmetry. Super-cool DM, instead, requires small values of the $B-L$ gauge coupling, such that we can assume that DM is charged under $B-L$, and thus automatically stable, compatibly with direct detection constraints.
} 
as well as electro-weak symmetry breaking and neutrino and DM masses,

$$
\frac{v}{w}=\sqrt{\frac{\lambda_{H S}}{2 \lambda_{H}}}, \quad m_{\nu}=-Y_{N}^{T} \cdot \frac{v^{2}}{M_{N}} \cdot Y_{N}, \quad M_{\mathrm{DM}}=M_{\phi}=\sqrt{\frac{\lambda_{S \phi}}{2}} w .
$$

We neglected the contribution of $\lambda_{H \phi}$ to $M_{\phi}$. Electroweak precision data imply the bound $M_{Z^{\prime}} / g_{B-L} \gtrsim 7 \mathrm{TeV}[80,81]$, up to corrections due to kinetic mixing. DM has $g_{\mathrm{DM}}=2$ degrees of freedom and is not destabilised by the symmetry breaking of the various gauge groups provided that $\lambda_{S \phi}, \lambda_{H \phi}, \lambda_{\phi}>0$ such that the DM scalar $\phi$ does not acquire a vacuum expectation value. The condition $\lambda_{\phi}>0$ is easily satisfied in view of the smaller $g_{B-L}^{4}$ contribution to its running:

$$
\beta_{\lambda_{\phi}}=\frac{1}{(4 \pi)^{2}}\left[6 g_{B-L}^{4}+2 \lambda_{H \phi}^{2}+\lambda_{S \phi}^{2}+20 \lambda_{\phi}^{2}-12 \lambda_{\phi} g_{B-L}^{2}\right] .
$$

The vacuum energy vanishes for $V_{\Lambda} \approx \beta_{\lambda_{S}} w^{4} / 16 \approx\left(3 M_{Z^{\prime}}^{4} / 8+M_{\mathrm{DM}}^{4} / 4\right) /(4 \pi)^{2}$ such that $T_{\mathrm{infl}} \approx\left(M_{Z^{\prime}}^{4}+2 M_{\mathrm{DM}}^{4} / 3\right)^{1 / 4} / 11$. The $s$ thermal potential is $V_{T} \approx T^{4}\left[3 f\left(M_{Z^{\prime}} / T\right)+\right.$ $\left.2 f\left(M_{\mathrm{DM}} / T\right)\right] / 2 \pi^{2}$ and its thermal mass is $M_{s}^{2 T}=\left(g_{B-L}^{2}+\lambda_{S \phi} / 12\right) T^{2}$, so that $T_{\text {end }}^{\mathrm{QCD}}=\langle h\rangle_{\mathrm{QCD}} \sqrt{\lambda_{H S} /\left(2 g_{B-L}^{2}+\lambda_{S \phi} / 6\right)}$.

The Spin-Independent cross section for DM direct detection receives two unavoidable contributions, from $Z^{\prime}$ mediation, and from $\lambda_{S \phi}$ (via the small mixing $\alpha \simeq-v / w$ between $h$ and $s$ ), as well as a contribution from $\lambda_{H \phi}$ :

$$
\sigma_{\mathrm{SI}} \approx \max \left(\frac{g_{B-L}^{4} q_{\phi}^{2} m_{N}^{2}}{4 \pi M_{Z^{\prime}}^{4}}, \frac{\lambda_{S \phi}^{2} m_{N}^{4} f^{2} \sin ^{2} 2 \alpha}{16 \pi M_{\mathrm{DM}}^{2} M_{s}^{4}}, \frac{\lambda_{H \phi}^{2} m_{N}^{4} f^{2}}{16 \pi M_{\mathrm{DM}}^{2} M_{h}^{4}}\right) .
$$

Finally, we need the DM pair production cross section that is at the origin of the subthermal population. In the non-relativistic limit, DM is produced in pairs in a $s$ wave way from two $Z^{\prime}$, from two scalars (possibly through a $Z^{\prime}$ ) while the production from two fermions via a $Z^{\prime}$ is $p$-wave suppressed. We get

$$
\begin{aligned}
\sigma_{\mathrm{ann}} v_{\mathrm{rel}} \approx & \frac{2 g_{B-L}^{4}}{\pi M_{\mathrm{DM}}^{2}} \operatorname{Re} \sqrt{1-\frac{M_{Z^{\prime}}^{2}}{M_{\mathrm{DM}}^{2}}}+ \\
& +\frac{\lambda_{H \phi}^{2} v^{2} \Gamma_{h}^{*} / M_{\mathrm{DM}}}{\left(4 M_{\mathrm{DM}}^{2}-M_{h}^{2}\right)^{2}+M_{h}^{2} \Gamma_{h}^{2}}+\frac{\lambda_{H \phi}^{2}}{64 \pi M_{\mathrm{DM}}^{2}} \operatorname{Re} \sqrt{1-\frac{M_{h}^{2}}{M_{\mathrm{DM}}^{2}}} \\
& +\frac{\lambda_{S \phi}^{2} w^{2} \Gamma_{s}^{*} / M_{\mathrm{DM}}}{\left(4 M_{\mathrm{DM}}^{2}-M_{s}^{2}\right)^{2}+M_{s}^{2} \Gamma_{s}^{2}}+\frac{\lambda_{S \phi}^{2}}{64 \pi M_{\mathrm{DM}}^{2}} \operatorname{Re} \sqrt{1-\frac{M_{s}^{2}}{M_{\mathrm{DM}}^{2}}} .
\end{aligned}
$$

where $\Gamma_{h, s}^{*}$ are the decay width into SM particles of a virtual $h, s$ with mass $2 M_{\mathrm{DM}} \cdot{ }^{6}$ We neglected the $s / h$ interference. These cross sections are similar to the ones in the DM scalar singlet model $[82,83]$.

We now have all the ingredients to compute the DM density. In figure 5 we plot it in the $\left(M_{Z^{\prime}}, g_{B-L}\right)$ plane, similarly to figure 3 for the $\mathrm{SU}(2)_{X}$ model. However the $\mathrm{U}(1)_{B-L}$

\footnotetext{
${ }^{6}$ Longitudinal components of $W^{ \pm}, Z$ enhance $\Gamma_{h}^{*} \simeq 3 M_{\mathrm{DM}}^{3} / 4 \pi v^{2}$ at $M_{\mathrm{DM}} \gg M_{h}$, such that $\sigma\left(\phi \phi^{*} \rightarrow\right.$ $\left.h^{*} \rightarrow W^{+} W^{-}, Z Z\right) \simeq 3 \sigma\left(\phi \phi^{*} \rightarrow h h\right)$ as demanded by $\mathrm{SU}(2)_{L}$ invariance.
} 

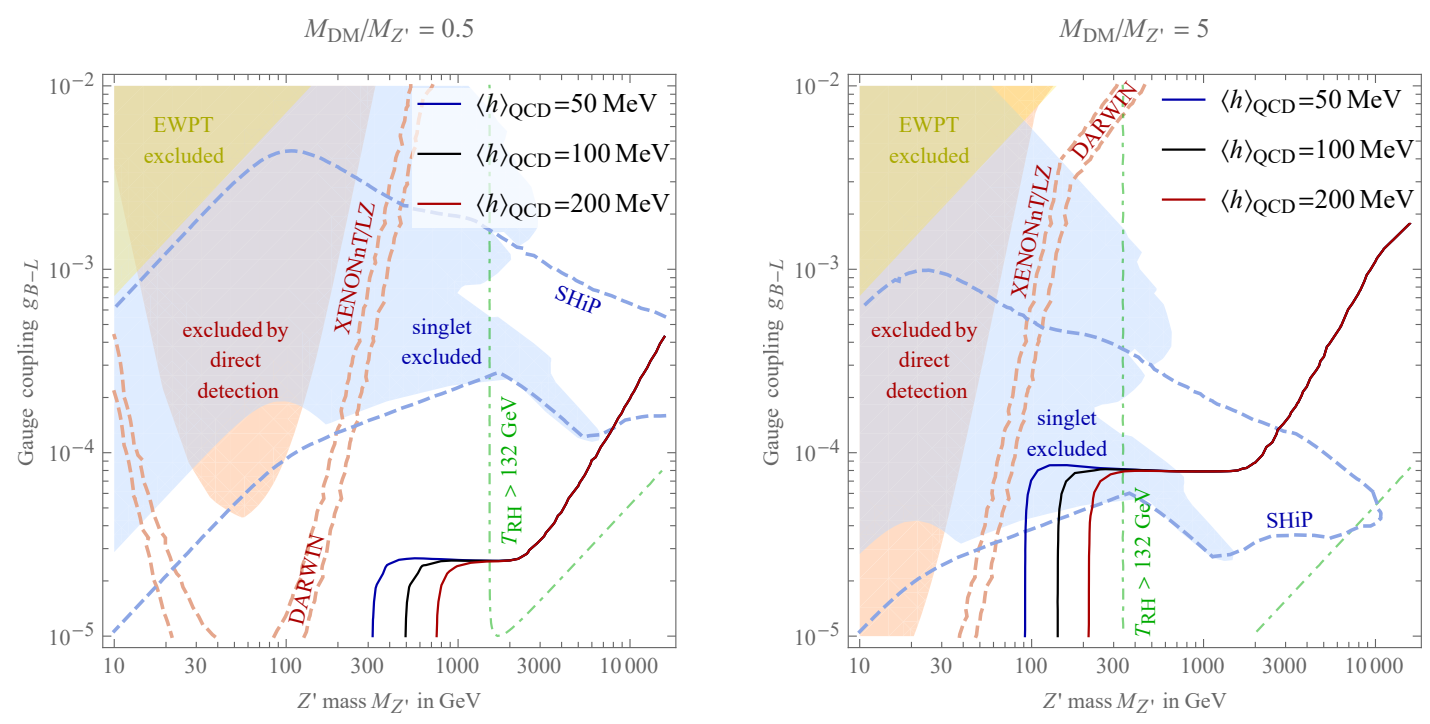

Figure 5. The observed DM abundance is reproduced along the solid curves, computed for different values of the uncertain QCD factor $\langle h\rangle_{\mathrm{QCD}}$. The region shaded in orange (blue) is excluded by direct DM searches (collider searches for the singlet $s$ ). The region shaded in yellow is excluded by precision data. Dashed curves indicate future detection prospects.

has a few extra free parameters, most importantly the DM mass. In figure 5 we thereby consider a few different values of the DM mass, and assume that the extra free parameters are in ranges which give neither enhancements nor cancellations in the various equations above. An important difference with respect to the previous model is that constraints from direct detection (in orange) are weaker. Baryogenesis through leptogenesis needs $T_{\mathrm{RH}} \gtrsim T_{\mathrm{sph}}$ : in the plotted parameter region this is satisfied when DM has a sizeable subthermal contribution, in addition to the super-cool contribution.

\section{Summary}

We presented a new mechanism that can reproduce the observed cosmological DM abundance when DM is a weak-scale particle. The mechanism arises in models where the weak scale is dynamically generated. The Universe remains trapped in a false vacuum where all particles are massless and undergoes a phase of thermal inflation during which all particles get diluted. This phase can be ended by the QCD phase transition or by vacuum decay to the true vacuum, where particles are massive. Light particles are regenerated in the subsequent reheating phase, but the DM abundance can remain suppressed, with a quite low temperature, due to supercooling. Figure 3 exemplifies the possible cosmological evolution. When super-cooling ends at $T \sim \Lambda_{\mathrm{QCD}}$, the desired DM abundance is obtained for weak-scale DM.

In section 3 we have shown that super-cool DM is produced in a simple model proposed in [10], where dynamical generation of the weak scale and DM stability is obtained adding to the $\mathrm{SM}$ a new $\mathrm{SU}(2)_{X}$ gauge group and a new scalar doublet. In section 4 we studied a model where the new gauge group is $\mathrm{U}(1)_{B-L}$. In both models DM is reproduced 
dominantly through super-cooling for DM masses of about $500 \mathrm{GeV}$ and for DM couplings of order $10^{-4}$ - smaller than in the freeze-out scenario, such that the simplest models of super-cool DM are still allowed by direct detection.

The $\mathrm{U}(1)_{B-L}$ gauge structure (and the scalar that breaks it), in addition of dynamically generating the symmetry breaking and of stabilizing DM, also gives rise to neutrino masses and to leptogenesis. This is a welcome feature, given that super-cooling erases a possibly pre-existing baryon asymmetry, which needs to be regenerated after reheating. Depending on the model and on its parameter space, the reheating temperature can be either larger or smaller than the decoupling temperature of weak sphalerons, such that the baryon asymmetry can be regenerated either through leptogenesis or through cold baryogenesis, possibly during the phase transition that ends super-cooling.

\section{Acknowledgments}

This work was supported by the ERC grant NEO-NAT. The work of T.H. and D.T. is supported by the Belgian FNRS-F.R.S., the "Probing DM with neutrinos" ULB-ARC grant, the "be.h" EOS grant n. 30820817, the IISN and a postdoctoral ULB fellowship. We thank Pasquale Serpico for clarifications about [22] and Christian Gross for discussions and suggestions about cold baryogenesis.

Open Access. This article is distributed under the terms of the Creative Commons Attribution License (CC-BY 4.0), which permits any use, distribution and reproduction in any medium, provided the original author(s) and source are credited.

\section{References}

[1] W.A. Bardeen, On naturalness in the standard model, FERMILAB-CONF-95-391 [INSPIRE]

[2] K.A. Meissner and H. Nicolai, Effective action, conformal anomaly and the issue of quadratic divergences, Phys. Lett. B 660 (2008) 260 [arXiv:0710.2840] [InSPIRE].

[3] M. Farina, D. Pappadopulo and A. Strumia, A modified naturalness principle and its experimental tests, JHEP 08 (2013) 022 [arXiv: 1303.7244] [INSPIRE].

[4] A.J. Helmboldt, P. Humbert, M. Lindner and J. Smirnov, Minimal conformal extensions of the Higgs sector, JHEP 07 (2017) 113 [arXiv:1603.03603] [INSPIRE].

[5] M. Shaposhnikov and A. Shkerin, Gravity, Scale Invariance and the Hierarchy Problem, arXiv: 1804.06376 [INSPIRE].

[6] A. Salvio and A. Strumia, Agravity, JHEP 06 (2014) 080 [arXiv:1403.4226] [InSPIRE].

[7] G.F. Giudice, G. Isidori, A. Salvio and A. Strumia, Softened Gravity and the Extension of the Standard Model up to Infinite Energy, JHEP 02 (2015) 137 [arXiv:1412.2769] [INSPIRE].

[8] G.M. Pelaggi, A. Strumia and S. Vignali, Totally asymptotically free trinification, JHEP 08 (2015) 130 [arXiv: 1507.06848] [INSPIRE].

[9] A. Salvio and A. Strumia, Agravity up to infinite energy, Eur. Phys. J. C 78 (2018) 124 [arXiv: 1705.03896] [INSPIRE]. 
[10] T. Hambye and A. Strumia, Dynamical generation of the weak and Dark Matter scale, Phys. Rev. D 88 (2013) 055022 [arXiv: 1306.2329] [INSPIRE].

[11] T. Hambye, Hidden vector dark matter, JHEP 01 (2009) 028 [arXiv:0811.0172] [INSPIRE].

[12] T. Hambye and M.H.G. Tytgat, Confined hidden vector dark matter, Phys. Lett. B 683 (2010) 39 [arXiv: 0907.1007] [INSPIRE].

[13] C. Arina, T. Hambye, A. Ibarra and C. Weniger, Intense Gamma-Ray Lines from Hidden Vector Dark Matter Decay, JCAP 03 (2010) 024 [arXiv:0912.4496] [INSPIRE].

[14] M. Bando, T. Kugo and K. Yamawaki, Nonlinear Realization and Hidden Local Symmetries, Phys. Rept. 164 (1988) 217 [INSPIRE].

[15] T. Konstandin and G. Servant, Cosmological Consequences of Nearly Conformal Dynamics at the TeV scale, JCAP 12 (2011) 009 [arXiv:1104.4791] [INSPIRE].

[16] P. Creminelli, A. Nicolis and R. Rattazzi, Holography and the electroweak phase transition, JHEP 03 (2002) 051 [hep-th/0107141] [INSPIRE].

[17] H. Davoudiasl, D. Hooper and S.D. McDermott, Inflatable Dark Matter, Phys. Rev. Lett. 116 (2016) 031303 [arXiv:1507.08660] [INSPIRE].

[18] A. Falkowski and J.M. No, Non-thermal Dark Matter Production from the Electroweak Phase Transition: Multi-TeV WIMPs and 'Baby-Zillas', JHEP 02 (2013) 034 [arXiv:1211.5615] [INSPIRE].

[19] J.E. Gunn, B.W. Lee, I. Lerche, D.N. Schramm and G. Steigman, Some Astrophysical Consequences of the Existence of a Heavy Stable Neutral Lepton, Astrophys. J. 223 (1978) 1015 [INSPIRE].

[20] J. Peñarrubia, Fluctuations of the gravitational field generated by a random population of extended substructures, Mon. Not. Roy. Astron. Soc. 474 (2018) 1482 [arXiv:1710.06443] [INSPIRE].

[21] E. Witten, Cosmological Consequences of a Light Higgs Boson, Nucl. Phys. B 177 (1981) 477 [INSPIRE].

[22] S. Iso, P.D. Serpico and K. Shimada, QCD-Electroweak First-Order Phase Transition in a Supercooled Universe, Phys. Rev. Lett. 119 (2017) 141301 [arXiv:1704.04955] [INSPIRE].

[23] T. Konstandin and G. Servant, Natural Cold Baryogenesis from Strongly Interacting Electroweak Symmetry Breaking, JCAP 07 (2011) 024 [arXiv:1104.4793] [INSPIRE].

[24] S. Bruggisser, B. Von Harling, O. Matsedonskyi and G. Servant, Electroweak Phase Transition and Baryogenesis in Composite Higgs Models, arXiv:1804.07314 [INSPIRE].

[25] G. Servant, Baryogenesis from Strong CP Violation and the QCD Axion, Phys. Rev. Lett. 113 (2014) 171803 [arXiv:1407.0030] [INSPIRE].

[26] A. Latosinski, A. Lewandowski, K.A. Meissner and H. Nicolai, Conformal Standard Model with an extended scalar sector, JHEP 10 (2015) 170 [arXiv:1507.01755] [INSPIRE].

[27] A. Tranberg and B. Wu, Cold Electroweak Baryogenesis in the Two Higgs-Doublet Model, JHEP 07 (2012) 087 [arXiv: 1203.5012] [INSPIRE].

[28] T. Hambye and M.H.G. Tytgat, Electroweak symmetry breaking induced by dark matter, Phys. Lett. B 659 (2008) 651 [arXiv:0707.0633] [INSPIRE]. 
[29] J.R. Espinosa, T. Konstandin, J.M. No and M. Quirós, Some Cosmological Implications of Hidden Sectors, Phys. Rev. D 78 (2008) 123528 [arXiv:0809.3215] [InSPIRE].

[30] R. Foot, A. Kobakhidze and R.R. Volkas, Stable mass hierarchies and dark matter from hidden sectors in the scale-invariant standard model, Phys. Rev. D 82 (2010) 035005 [arXiv: 1006.0131] [INSPIRE].

[31] R. Foot and A. Kobakhidze, Electroweak Scale Invariant Models with Small Cosmological Constant, Int. J. Mod. Phys. A 30 (2015) 1550126 [arXiv:1112.0607] [InSPIRE].

[32] K. Ishiwata, Dark Matter in Classically Scale-Invariant Two Singlets Standard Model, Phys. Lett. B 710 (2012) 134 [arXiv:1112.2696] [INSPIRE].

[33] A. Goudelis, B. Herrmann and O. Stål, Dark matter in the Inert Doublet Model after the discovery of a Higgs-like boson at the LHC, JHEP 09 (2013) 106 [arXiv:1303.3010] [INSPIRE].

[34] T.G. Steele, Z.-W. Wang, D. Contreras and R.B. Mann, Viable dark matter via radiative symmetry breaking in a scalar singlet Higgs portal extension of the standard model, Phys. Rev. Lett. 112 (2014) 171602 [arXiv:1310.1960] [INSPIRE].

[35] C.D. Carone and R. Ramos, Classical scale-invariance, the electroweak scale and vector dark matter, Phys. Rev. D 88 (2013) 055020 [arXiv: 1307.8428] [INSPIRE].

[36] A. Farzinnia, H.-J. He and J. Ren, Natural Electroweak Symmetry Breaking from Scale Invariant Higgs Mechanism, Phys. Lett. B 727 (2013) 141 [arXiv:1308.0295] [INSPIRE].

[37] V.V. Khoze, Inflation and Dark Matter in the Higgs Portal of Classically Scale Invariant Standard Model, JHEP 11 (2013) 215 [arXiv:1308.6338] [INSPIRE].

[38] E. Gabrielli, M. Heikinheimo, K. Kannike, A. Racioppi, M. Raidal and C. Spethmann, Towards Completing the Standard Model: Vacuum Stability, EWSB and Dark Matter, Phys. Rev. D 89 (2014) 015017 [arXiv: 1309.6632] [INSPIRE].

[39] J. Guo and Z. Kang, Higgs Naturalness and Dark Matter Stability by Scale Invariance, Nucl. Phys. B 898 (2015) 415 [arXiv:1401.5609] [INSPIRE].

[40] S. Benic and B. Radovcic, Electroweak breaking and Dark Matter from the common scale, Phys. Lett. B 732 (2014) 91 [arXiv:1401.8183] [InSPIRE].

[41] V.V. Khoze, C. McCabe and G. Ro, Higgs vacuum stability from the dark matter portal, JHEP 08 (2014) 026 [arXiv: 1403.4953] [INSPIRE].

[42] A. Farzinnia and J. Ren, Higgs Partner Searches and Dark Matter Phenomenology in a Classically Scale Invariant Higgs Boson Sector, Phys. Rev. D 90 (2014) 015019 [arXiv: 1405.0498] [INSPIRE].

[43] G.M. Pelaggi, Predictions of a model of weak scale from dynamical breaking of scale invariance, Nucl. Phys. B 893 (2015) 443 [arXiv:1406.4104] [InSPIRE].

[44] A. Farzinnia and J. Ren, Strongly First-Order Electroweak Phase Transition and Classical Scale Invariance, Phys. Rev. D 90 (2014) 075012 [arXiv: 1408.3533] [INSPIRE].

[45] W. Altmannshofer, W.A. Bardeen, M. Bauer, M. Carena and J.D. Lykken, Light Dark Matter, Naturalness and the Radiative Origin of the Electroweak Scale, JHEP 01 (2015) 032 [arXiv: 1408.3429] [INSPIRE].

[46] S. Benic and B. Radovcic, Majorana dark matter in a classically scale invariant model, JHEP 01 (2015) 143 [arXiv:1409.5776] [INSPIRE]. 
[47] J. Guo, Z. Kang, P. Ko and Y. Orikasa, Accidental dark matter: Case in the scale invariant local B-L model, Phys. Rev. D 91 (2015) 115017 [arXiv:1502.00508] [INSPIRE].

[48] Z. Kang, View FImP miracle (by scale invariance) à la self-interaction, Phys. Lett. B 751 (2015) 201 [arXiv: 1505.06554] [InSPIRE].

[49] K. Endo and K. Ishiwata, Direct detection of singlet dark matter in classically scale-invariant standard model, Phys. Lett. B 749 (2015) 583 [arXiv:1507.01739] [INSPIRE].

[50] A.D. Plascencia, Classical scale invariance in the inert doublet model, JHEP 09 (2015) 026 [arXiv: 1507.04996] [INSPIRE].

[51] A. Ahriche, K.L. McDonald and S. Nasri, A Radiative Model for the Weak Scale and Neutrino Mass via Dark Matter, JHEP 02 (2016) 038 [arXiv: 1508.02607] [InSPIRE].

[52] A. Karam and K. Tamvakis, Dark matter and neutrino masses from a scale-invariant multi-Higgs portal, Phys. Rev. D 92 (2015) 075010 [arXiv:1508.03031] [INSPIRE].

[53] V.V. Khoze and A.D. Plascencia, Dark Matter and Leptogenesis Linked by Classical Scale Invariance, JHEP 11 (2016) 025 [arXiv:1605.06834] [INSPIRE].

[54] A. Karam and K. Tamvakis, Dark Matter from a Classically Scale-Invariant SU(3) $X$, Phys. Rev. D 94 (2016) 055004 [arXiv: 1607.01001] [INSPIRE].

[55] M. Heikinheimo, T. Tenkanen and K. Tuominen, WIMP miracle of the second kind, Phys. Rev. D 96 (2017) 023001 [arXiv: 1704.05359] [INSPIRE].

[56] J.R. Espinosa and M. Quirós, Novel Effects in Electroweak Breaking from a Hidden Sector, Phys. Rev. D 76 (2007) 076004 [hep-ph/0701145] [INSPIRE].

[57] A. Joti et al., (Higgs) vacuum decay during inflation, JHEP 07 (2017) 058 [arXiv: 1706.00792] [INSPIRE].

[58] P. Petreczky, Lattice QCD at non-zero temperature, J. Phys. G 39 (2012) 093002 [arXiv: 1203.5320] [INSPIRE].

[59] Particle Data Group collaboration, C. Patrignani et al., Review of Particle Physics, Chin. Phys. C 40 (2016) 100001 [INSPIRE].

[60] J. Braun and H. Gies, Chiral phase boundary of QCD at finite temperature, JHEP 06 (2006) 024 [hep-ph/0602226] [INSPIRE].

[61] M. Quirós, Finite temperature field theory and phase transitions, in Proceedings, Summer School in High-energy physics and cosmology, Trieste, Italy, June 29-July 17, 1998, pp. 187-259 (1999) [hep-ph/9901312] [INSPIRE].

[62] P.W. Graham, J. Mardon and S. Rajendran, Vector Dark Matter from Inflationary Fluctuations, Phys. Rev. D 93 (2016) 103520 [arXiv:1504.02102] [INSPIRE].

[63] Y. Bai and A.J. Long, Six Flavor Quark Matter, JHEP 06 (2018) 072 [arXiv:1804.10249] [INSPIRE].

[64] XENON collaboration, E. Aprile et al., First Dark Matter Search Results from the XENON1T Experiment, Phys. Rev. Lett. 119 (2017) 181301 [arXiv:1705.06655] [INSPIRE].

[65] XENON collaboration, E. Aprile et al., Physics reach of the XENON1T dark matter experiment, JCAP 04 (2016) 027 [arXiv: 1512.07501] [INSPIRE].

[66] LZ collaboration, D.S. Akerib et al., LUX-ZEPLIN (LZ) Conceptual Design Report, arXiv: 1509.02910 [INSPIRE]. 
[67] DARWIN collaboration, J. Aalbers et al., DARWIN: towards the ultimate dark matter detector, JCAP 11 (2016) 017 [arXiv: 1606.07001] [INSPIRE].

[68] SHIP collaboration, G. Lanfranchi, Sensitivity of the SHiP experiment to a light scalar particle mixing with the Higgs, CERN-SHiP-NOTE-2017-001.

[69] SHIP collaboration, M. Anelli et al., A facility to Search for Hidden Particles (SHiP) at the CERN SPS, arXiv:1504.04956 [INSPIRE].

[70] A. Pilaftsis, CP violation and baryogenesis due to heavy Majorana neutrinos, Phys. Rev. D 56 (1997) 5431 [hep-ph/9707235] [INSPIRE].

[71] A. Pilaftsis and T.E.J. Underwood, Resonant leptogenesis, Nucl. Phys. B 692 (2004) 303 [hep-ph/0309342] [INSPIRE].

[72] P.S. Bhupal Dev, P. Millington, A. Pilaftsis and D. Teresi, Flavour Covariant Transport Equations: an Application to Resonant Leptogenesis, Nucl. Phys. B 886 (2014) 569 [arXiv: 1404.1003] [INSPIRE].

[73] E.K. Akhmedov, V.A. Rubakov and A.Yu. Smirnov, Baryogenesis via neutrino oscillations, Phys. Rev. Lett. 81 (1998) 1359 [hep-ph/9803255] [INSPIRE].

[74] T. Asaka and M. Shaposhnikov, The nuMSM, dark matter and baryon asymmetry of the universe, Phys. Lett. B 620 (2005) 17 [hep-ph/0505013] [INSPIRE].

[75] T. Hambye and D. Teresi, Higgs doublet decay as the origin of the baryon asymmetry, Phys. Rev. Lett. 117 (2016) 091801 [arXiv: 1606.00017] [INSPIRE].

[76] T. Hambye and D. Teresi, Baryogenesis from L-violating Higgs-doublet decay in the density-matrix formalism, Phys. Rev. D 96 (2017) 015031 [arXiv:1705.00016] [INSPIRE].

[77] J.-M. Frere, T. Hambye and G. Vertongen, Is leptogenesis falsifiable at LHC?, JHEP 01 (2009) 051 [arXiv: 0806. 0841] [inSPIRE].

[78] J. Heeck and D. Teresi, Leptogenesis and neutral gauge bosons, Phys. Rev. D 94 (2016) 095024 [arXiv: 1609.03594] [INSPIRE].

[79] S. Iso, N. Okada and Y. Orikasa, The minimal B-L model naturally realized at TeV scale, Phys. Rev. D 80 (2009) 115007 [arXiv:0909.0128] [INSPIRE].

[80] G. Cacciapaglia, C. Csáki, G. Marandella and A. Strumia, The Minimal Set of Electroweak Precision Parameters, Phys. Rev. D 74 (2006) 033011 [hep-ph/0604111] [InSPIRE].

[81] E. Salvioni, G. Villadoro and F. Zwirner, Minimal Z-prime models: Present bounds and early LHC reach, JHEP 11 (2009) 068 [arXiv:0909.1320] [INSPIRE].

[82] J. McDonald, Gauge singlet scalars as cold dark matter, Phys. Rev. D 50 (1994) 3637 [hep-ph/0702143] [INSPIRE].

[83] C.P. Burgess, M. Pospelov and T. ter Veldhuis, The Minimal model of nonbaryonic dark matter: A Singlet scalar, Nucl. Phys. B 619 (2001) 709 [hep-ph/0011335] [INSPIRE]. 